MYU Tokyo

S \& M 1163

\title{
Parametrically Actuated Resonant Micromirror Using Stiffness Tunable Torsional Springs
}

\author{
Yusuke Kawai, Jin-Hyeok Kim, Naoki Inomata, ${ }^{1}$ and Takahito Ono* \\ Graduate School of Engineering, Tohoku University, \\ 6-6-01 AzaAoba, Aramaki, Aoba-ku, Sendai 980-8579, Japan \\ ${ }^{1}$ Microsystem Integration Center, Graduate School of Engineering, Tohoku University, \\ 6-6-01 AzaAoba, Aramaki, Aoba-ku, Sendai 980-8579, Japan
}

(Received August 31, 2015; accepted October 28, 2015)

Keywords: micromirror, parametric amplification, hard spring effect, frequency tuning, torsional spring

In this study, we design, fabricate and evaluate a resonant micromirror that can amplify the vibration amplitude by mechanical parametric amplification. It is shown that the spring constant of torsion bars supporting the micromirror can be varied by stress generated using an electrothermal actuator, which can tune the resonant frequency of the torsional mode. The parametric amplification of the vibration of the torsional mode is demonstrated by applying a pumping signal using the electrothermal actuator in addition to a driving signal. As the driving power increases, the resonant frequency increases owing to the nonlinear spring effect of the torsional spring. However, the frequency shift can be compensated for by applying stress to the torsional spring using the frequency tuning mechanism for the reliable operation of the micromirror.

\section{Introduction}

Micromirror scanners are one of successful microelectromechanical devices, and have been used for various applications, ${ }^{(1)}$ including display, ${ }^{(2)}$ endoscopic imaging, ${ }^{(3)}$ optical switches, ${ }^{(4)}$ and barcode readers, ${ }^{(5)}$ owing to their small size and low power consumption. Although micromirrors have a wide range of potential applications, their use is limited owing to the performance limitation and reliability. For example, for the applications of a range finder for a robot vision and a car safety system, a micromirror with a large scanning angle is required to achieve a wide area of view. Large rotation angle, low power consumption, and low driving voltage are key performance characteristics of micromirrors. Various micromirror scanners with electrostatic, electromagnetic, thermal and piezoelectric actuators have been developed to satisfy those requirements. ${ }^{(6)}$ Micromirror scanners are often operated in resonance to increase the scanning angle of the mirror; however, the achievable scanning angle is still limited owing to the limited actuation force. To increase the amplitude of a resonator, mechanical parametric amplification was performed in a cantilever structure. (7,8) Parametric amplification requires the additional modulation (pumping) of the spring with a modulation frequency $n$-times higher than the fundamental resonant frequency of the resonator, where $n$ is an integer $(n=2,3,4, \ldots)$. In addition, the amplification gain depends on the phase difference between the actuation and pumping signals. In this paper, we propose a parametrically driven micromirror scanner based on a frequency tuning mechanism (bent beam ${ }^{\bar{*}}$ Corresponding author: e-mail: ono@nme.mech.tohoku.ac.jp 
actuator) that changes the spring constant of the micromirror scanner in order to amplify the scanning angle. As an additional effect of resonant actuation, the hard spring effect due to the nonlinearity of the spring is induced, ${ }^{(9)}$ where the resonant frequency tends to shift to a higher value at a lager scanning angle owing to tension. This undesired frequency shift makes the control of the vibration amplitude difficult. The integrated actuator for tuning the spring constant can possibly prevent this change in resonant frequency.

The design, fabrication and evaluation of the resonant micromirror scanner having a torsion bar with a tunable spring are presented. Also, the tuning of the resonant frequency and the parametric amplification of scanning are demonstrated using the fabricated micromirror with the spring tuning mechanism. An integrated electrothermal actuator can push the torsion bar of the mirror to apply force, which can tune the spring constant in the torsional mode.

\section{Concept of Parametric Amplification and Tunable Spring}

To achieve the parametric amplification of scanning angle, a pumping signal must be applied to the torsional spring in addition to the actuation force. If the motion is supposed to be onedimensional, the pumping signal is twice the resonant frequency. This behavior is described by the Newton equation ${ }^{(8)}$

$$
m \frac{d^{2} x}{d t^{2}}+\frac{m \omega_{0}}{Q} \frac{d x}{d t}+\left[k_{0}+\Delta k \sin 2 \omega_{0} t\right] x=F_{0} \cos \left(\omega_{0} t+\phi\right),
$$

where $x$ is the displacement, $m$ is the effective mass of the mass-spring system, $Q$ is the mechanical quality factor, $k_{0}$ is the original spring constant, $\Delta k$ is the modulation amplitude of the spring constant, $\phi$ is the phase difference between the driving signal and the pumping signal, $\omega_{0}$ is the resonant frequency of the resonator, and $F_{0}$ is the amplitude of the driving force. The gain $G$, which is defined by the amplitude with parametric amplification against the amplitude without parametric amplification, is given by

$$
G=\sqrt{\left(\frac{\cos \phi}{1+\frac{Q \Delta k}{2 k_{0}}}\right)^{2}\left(\frac{\sin \phi}{1-\frac{Q \Delta k}{2 k_{0}}}\right)^{2}} .
$$

From Eq. (2), the maximum $G$ is obtained at $\phi=\pi / 2$.

The shape of the torsion bar of a micromirror was modified, and feedback control actuation was performed to tune the resonant frequency. ${ }^{(10,11)}$ Moreover, it is known that the resonant frequency increases with increasing tension generated by the stress of torsion bars. ${ }^{(12)}$ The resonant frequency of rotating mirrors is governed by the moment of inertia and the torsional spring, and compressive force makes the torsional spring soft. In order to modulate the spring constant of the mirror scanners suspended with the torsion bars, a tuning actuator is integrated, which varies the tension in the torsion bars along the bar direction. 


\section{Design and Fabrication}

The schematic image of the proposed micromirror scanner with a tunable spring mechanism is shown in Fig. 1. The micromirror scanner consists of a cylindrical mirror and a tunable torsional spring. The micromirror is suspended by torsional bars connected with a thermally driven bent beam actuator. ${ }^{(13)}$ Because the thermal actuator can generate a large force, the tensions of the torsional bars can be markedly changed. This micromirror can be oscillated by an external lead zirconate titanate (PZT) actuator. The bent beam actuator can be deformed by heat generated by flowing a current into the beam between two electrodes. When the current flows into the bent beam actuator, this actuator gives a compressive stress to the torsional bar of the micromirror because of the thermal expansion of the bent beam actuator. This compressive stress decreases the spring constant and the resonant frequency of this micromirror. This spring tuning mechanism is used as a pumping actuator for parametric amplification, also used for tuning the resonant frequency.

The micromirror scanner is fabricated by using a conventional microfabrication technique. The micromirror scanner is made of single-crystal silicon because of its excellent mechanical properties. The fabrication process is shown in Fig. 2. The starting material is a silicon-oninsulator wafer, which has a resistivity of $0.02 \Omega \cdot \mathrm{cm}$. It also consists of device and handle layers with thicknesses of 80 and $350 \mu \mathrm{m}$, respectively. A 2- $\mu \mathrm{m}$-thick buried oxide layer exists between the device layer and the handle layer. As the first step, $\mathrm{Cr}$ and Au films with thicknesses of 30 and $150 \mathrm{~nm}$, respectively, are deposited and patterned to form the mirror and electrode pattern by a lift-off process (step 2). Deep reactive ion etching (DRIE) is performed to form the mirror shape, torsional bars and bent beam actuator with a depth of $80 \mu \mathrm{m}$ from the top side of the substrate (step 3). Then, DRIE is performed from the back surface to form the proof mass above the mirror. A buried oxide layer is etched in hydrofluoric acid to release the scanner structure (step 4). After the release

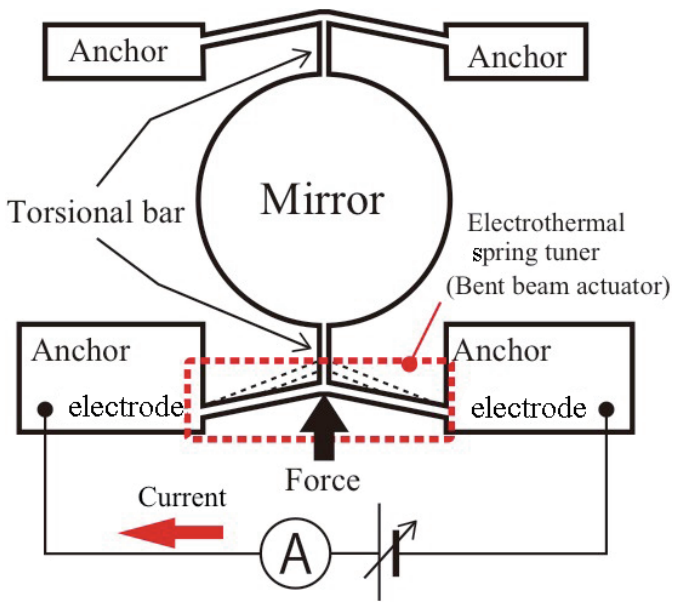

Fig. 1. (Color online) Schematic shows the concept of a spring tuner integrated with micromirror scanner. Electrothermally driven bent-beam actuator pushes torsional bars to generate compressive force, which can tune the torsional spring.

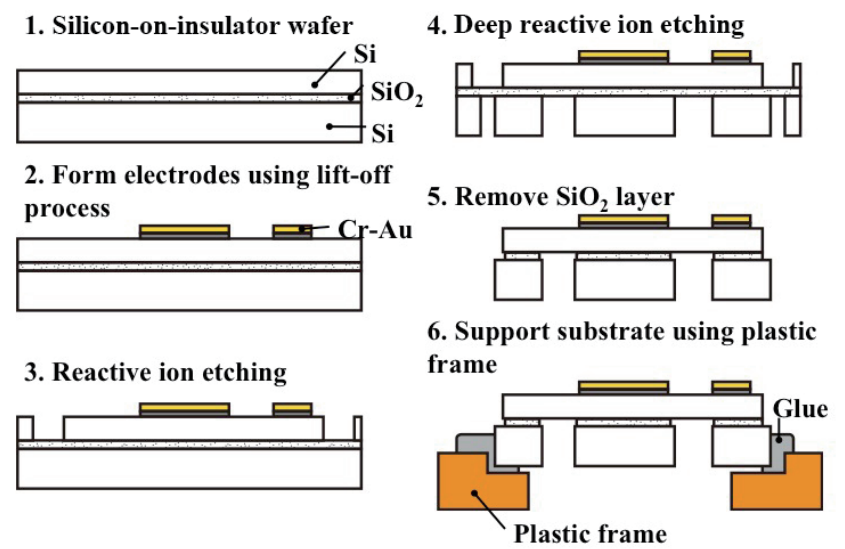

Fig. 2. (Color online) Sequence of fabrication process for the micromirror scanner. In steps 5 and 6 , the micromachined scanner is diced for easier separation and supported by a plastic frame for easier release. 
process the micromirror scanner was bonded to the plastic frame using glue to support and form the gap below the mirror.

Figure 3 shows the scanning electron microscopy image of the fabricated micromirror scanner. The inset of Fig. 3 shows the magnified image of the bent beam actuator. The dimensions of the micromirror are summarized in Table 1. The fundamental resonant frequency of the torsional mode of the mirror and the $Q$ factor are $723 \mathrm{~Hz}$ and 7000 , respectively.

\section{Experimental Method}

The performance characteristics of the tuning effect due to the additional compressive force provided by the integrated bent beam actuator and the amplification effect for scanning angle with parametric actuation using the spring modulation are evaluated through the fabricated micromirror scanner. Figure 4 shows the experimental setup for the measurement of the optical scanning angle in the torsional mode. The fabricated micromirror is mounted on a piezoceramic actuator to vibrate the micromirror. The piezoceramic and bent beam actuators are electrically connected to function generators, which can output driving and pumping signals with arbitrary amplitude and phase difference, respectively. The optical scanning angle is measured from the trajectory length of the laser beam reflected by the mirror. The measured fundamental resonant frequency of the fabricated micromirror in the torsional mode is $723.05 \mathrm{~Hz}$. The mechanical $Q$ factor of the scanner is approximately 7000. For the measurements of the tuning effect, the bent beam actuator is driven by dc voltage application, and the driving frequency for mirror vibration is swept upward. For parametric actuation, the piezoceramic actuator is driven at the resonant frequency $f_{0}$ of the micromirror for actuation, and also the bent beam actuator is driven at the same frequency by flowing a current with a frequency $f_{0}$ because the thermal actuator generates force through Joule heating with twofold driving frequency. Thus, the pumping frequency for parametric amplification will be $2 f_{0}$. The phase difference $\phi$ is swept to measure the parametric amplification gain characteristics.

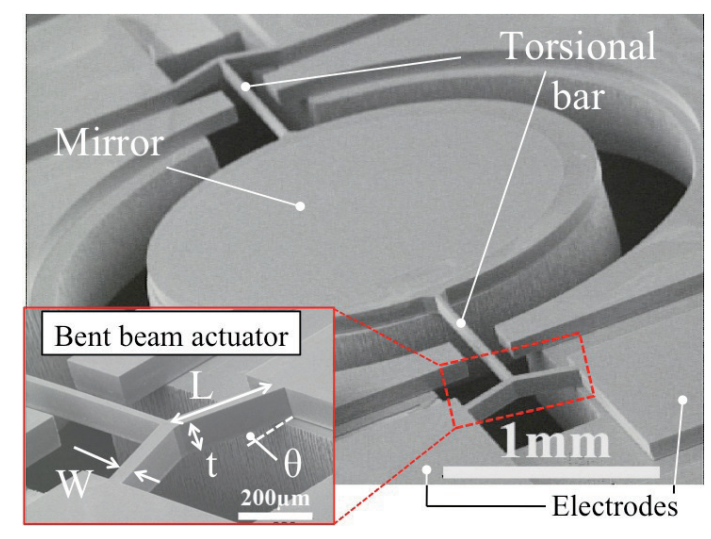

Fig. 3. (Color online) Scanning electron microscopy image of fabricated micromirror scanner. The inset shows an integrated bent beam actuator as a compression-type spring tuner.
Table 1

Dimensions of the micromirror scanner.

\begin{tabular}{|c|c|c|c|}
\hline \multicolumn{4}{|l|}{ Mirror } \\
\hline \multicolumn{2}{|c|}{ Diameter } & \multicolumn{2}{|c|}{ Thickness } \\
\hline \multicolumn{2}{|c|}{$3000 \mu \mathrm{m}$} & \multicolumn{2}{|c|}{$430 \mu \mathrm{m}$} \\
\hline \multicolumn{4}{|c|}{ Torsional bar } \\
\hline \multicolumn{2}{|l|}{ Length } & th & Thickness \\
\hline \multicolumn{2}{|l|}{$1000 \mu \mathrm{m}$} & $\mathrm{am}$ & $80 \mu \mathrm{m}$ \\
\hline \multicolumn{4}{|c|}{ Bent beam actuator } \\
\hline $\begin{array}{l}\text { Length: } L \\
260 \mu \mathrm{m}\end{array}$ & $\begin{array}{l}\text { Width: } W \\
50 \mu \mathrm{m}\end{array}$ & $\begin{array}{c}\text { Thickness: } t \\
80 \mu \mathrm{m}\end{array}$ & $\begin{array}{c}\text { Angle: } \theta \\
22.6^{\circ}\end{array}$ \\
\hline
\end{tabular}




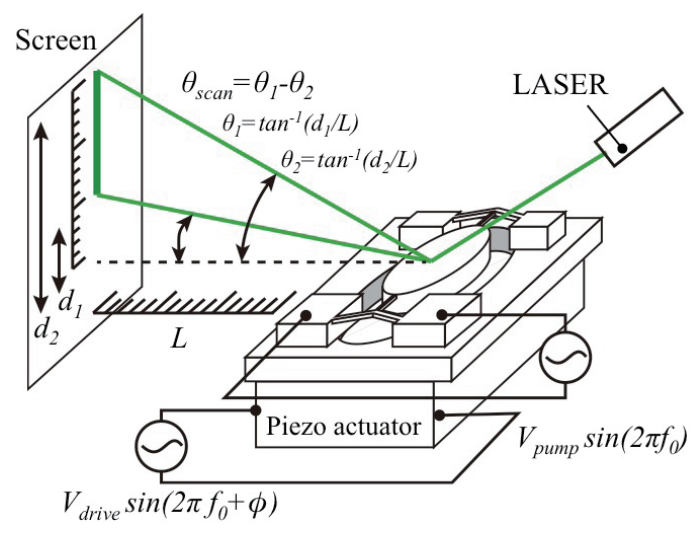

Fig. 4. (Color online) Schematic figure of the experimental setup for the measurement of optical scanning and parametric amplification.

\section{Frequency Tuning and Parametric Amplification}

First, we investigate the tuning range of resonant frequency using the thermoelectric bent beam actuator. The electrical resistance of the actuator without power input is approximately $205 \Omega$. As the input dc power to the actuator increases, the resistance decreases owing to Joule heating as shown in Fig. 5. Also, the measured resonant frequency in the fundamental torsional mode decreases with increasing power. The temperature of the bent beam actuator increases with increasing current flow owing to heat generation, and the thermal expansion generates the driving force applied to the torsional beam. Thus, the resonant frequency markedly decreases with increasing input power to the bent beam actuator. The maximum changing ratio of the resonant frequencies before and after the actuation of the bent beam actuator is 0.59 at the input power of $1.1 \mathrm{~W}$. The corresponding variation in torsional spring constant is approximately $35 \%$ compared with the initial value because the variation of the torsional spring is given by the square of the resonant frequency variation, i.e., $\sim 0.59^{2}$. By applying a power of $1.17 \mathrm{~W}(9 \mathrm{~V}, 130 \mathrm{~mA})$, buckling was observed in torsion bars owing to the excess additional compressive force from the bent beam actuator.

Figure 6 shows the phase dependence on the gain of the parametric amplification of the micromirror at a driving voltage of $10 V_{\mathrm{pp}}$ and a pumping ac voltage of $10 V_{\mathrm{pp}}$. Here, the experiments are performed at a driving frequency of $723.05 \mathrm{~Hz}$, and also the pumping frequency is set to a value twice the driving frequency. It can be seen that the amplification characteristic depends on the phase difference $\phi$. This result shows good agreement with the theory of parametric amplification shown in Eq. (3), explaining the parametric amplification of the resonator. Theoretically, the phase indicating maximum scanning amplitude will be 90 degrees, but in the experiment, the maximum value is observed at a phase of approximately 0 degrees. This is possibly due to the thermal response delay of the thermoelectric actuator. The thermal time constant $\tau$ of the bent beam actuator is approximated by $\tau=R_{\mathrm{th}} C_{\mathrm{th}}$, where $R_{\mathrm{th}}$ is the thermal resistance of the actuator beams and $C_{\mathrm{th}}$ is the thermal capacitance of the actuator beams. Also, the thermal resistance is given by $R_{\mathrm{th}}=L / \kappa A$, where $L$ is the length of the beam, $A$ is the cross section of the beam, and $\kappa$ is the thermal conductivity $(149 \mathrm{~W} / \mathrm{mK}$ for $\mathrm{Si})$. Thermal capacitance is given by $C_{\mathrm{th}}=c \rho h$, where $c$ 


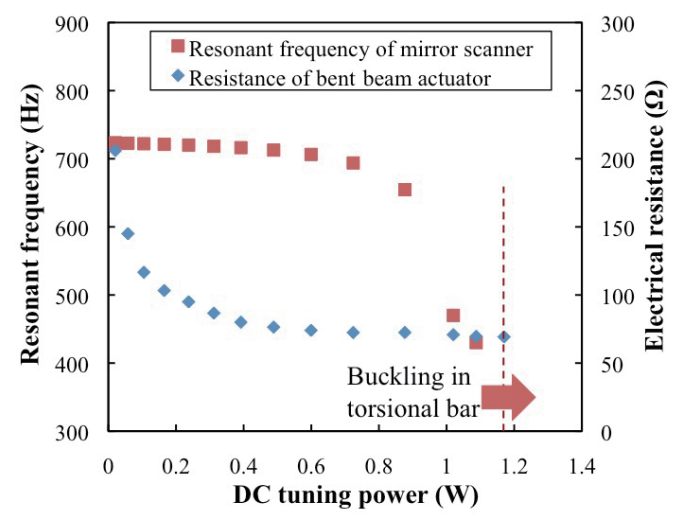

Fig. 5. (Color online) Input tuning power dependence on the resonant frequency of fundamental torsional mode in micromirror and electrical resistance of bent beam actuator. Buckling was observed in the torsional bar of the micromirror with an input power above $1.17 \mathrm{~W}$.

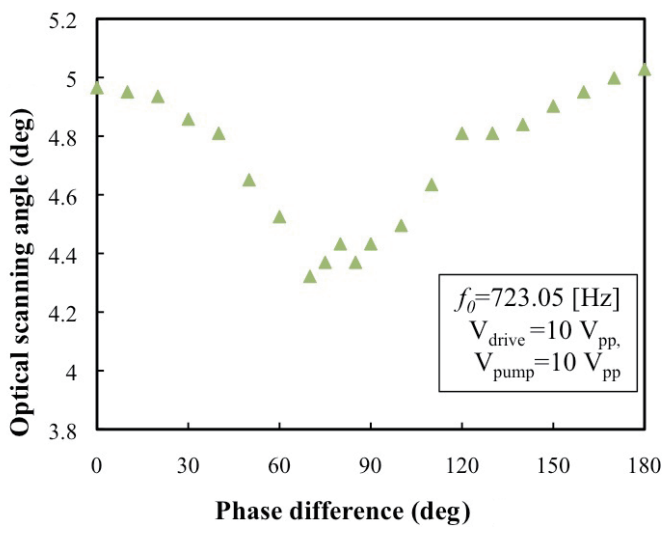

Fig. 6. (Color online) Gain and squeeze characteristics of parametric amplification at a resonant frequency of $723.05 \mathrm{~Hz}$.

is the specific heat $(735 \mathrm{~J} / \mathrm{kgK}$ for $\mathrm{Si}), \rho$ is the density $\left(2330 \mathrm{~kg} / \mathrm{m}^{3}\right.$ for $\left.\mathrm{Si}\right)$ and $V$ is the volume of the beam. From the dimensions $\left(L=260 \mu \mathrm{m}, A=80 \times 50 \mu \mathrm{m}^{2}\right)$ of the bent beam actuator, the time constant is calculated to be $7.8 \times 10^{-4}$, which corresponds to a frequency $(1 / 2 \pi \tau)$ of approximately $200 \mathrm{~Hz}$. Thus, this low thermal response time of the actuator causes the delay and also decreases the pumping efficiency for parametric amplification.

At 0 degrees in phase difference between driving and pumping signals, the maximum gain is $0.8 \mathrm{~dB}$. The vibration of the micromirror could not be observed when only the pumping actuator is driven without the driving signal.

Figure 7 shows the relationship between the optical scanning angle and the pumping signal when a driving voltage of $20 \mathrm{~V}$ with $\phi=0$ is applied. The driving power and pumping frequency were adjusted to find the maximum scanning angle of the micromirror. With increasing amplitude of the pumping voltage, the optical scanning angle also increases. A maximum scanning angle of 30 degrees is obtained at a pumping voltage of $20 V_{\mathrm{pp}}$, which corresponds to a $9 \mathrm{~dB}$ gain. From the result of the frequency tuning range shown in Fig. 5 and the amplification characteristic, the tuning range of the torsional spring is strongly related to the maximum amplification gain for the enhancement of scanning angle.

\section{Amplitude Control Using Tuning Mechanism}

For some applications of micromirrors operated in the resonant mode, a large scanning angle is required. The scanning angle is still limited owing to a nonlinear spring effect known as the hard spring effect. This undesired frequency shift makes the control of the vibration amplitude difficult. We propose the actuation method based on a tuning actuator for a reliable actuation. As shown in Fig. 8, the hard spring effect shifts the resonant frequency as the scanning angle increases; therefore, the achievable scanning angle of the micromirror decreases at a given power. Frequency tuning can compensate for the frequency shift and can amplify the scanning angle. 


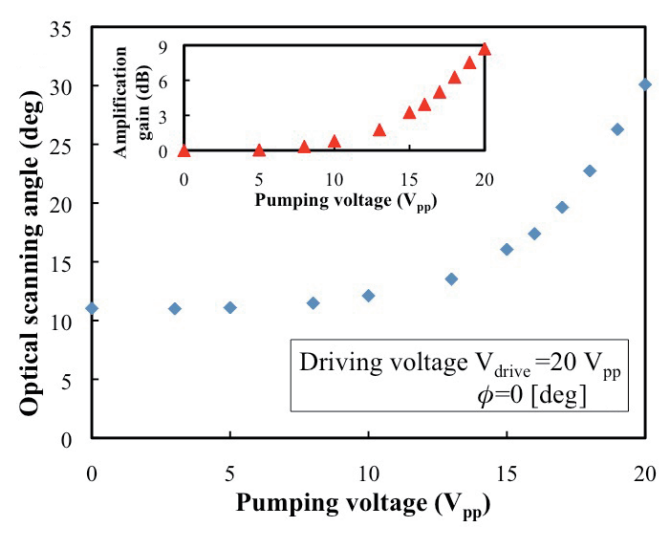

Fig. 7. (Color online) Pumping voltage dependence on optical scanning angle with the driving voltage of $20 V_{\mathrm{pp}}$ and phase difference of 0 degrees. The inset shows the corresponding gain characteristics.
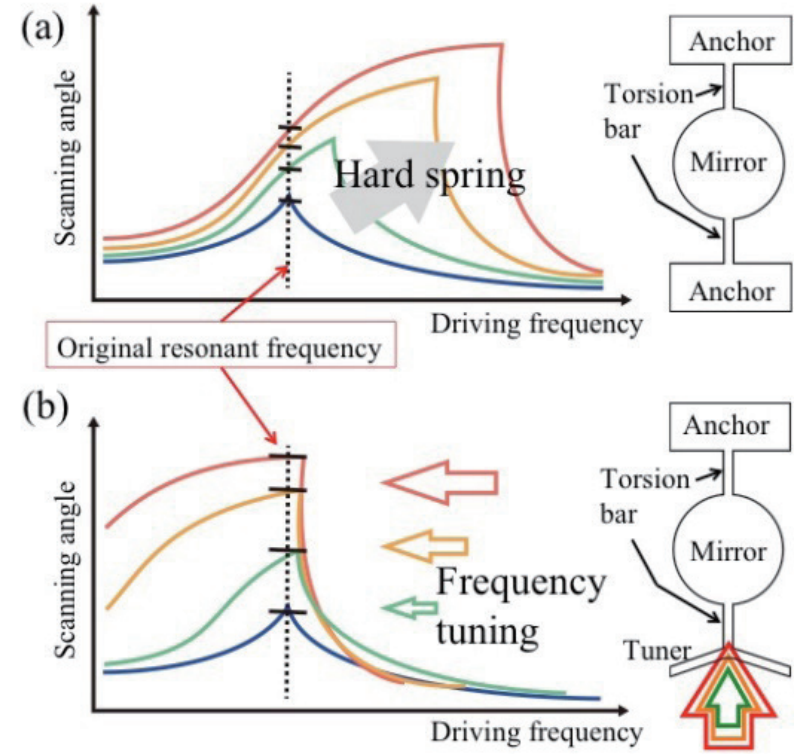

Fig. 8. (Color online) Schematic concept of frequency compensation.

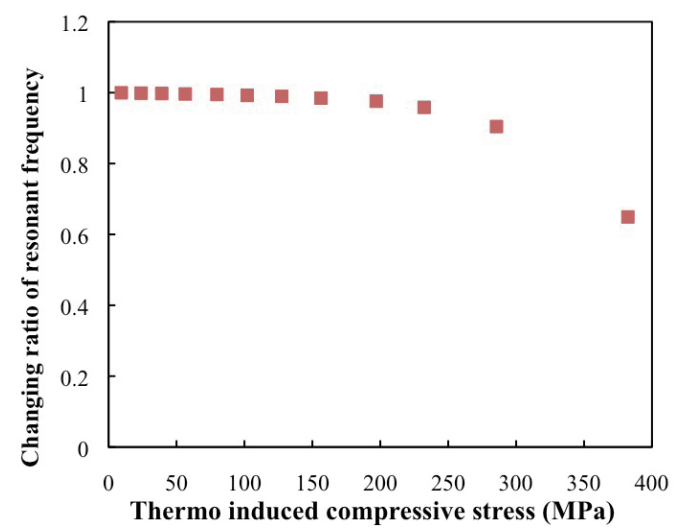

Fig. 9. (Color online) Characteristic of frequency changing due to the thermoelectrically induced compressive stress.

The performance of the tuning effect due to the additional compressive force provided by the integrated bent beam actuator is evaluated using the fabricated micromirror. The piezoceramic actuator mounting the micromirror is driven by applying a constant ac voltage using a function generator. For the measurements of the tuning effect, the bent beam actuator is driven by the application of a dc voltage, and the driving frequency for mirror vibration is swept. The resonant frequency in the torsional mode is markedly changed by applying an induced compressive force among the torsion bars, as shown in Fig. 9. The temperature of the bent beam actuator is measured by thermography, and the compressive stress is estimated from thermal stress based on the measured temperature. When the applied compressive force is $390 \mathrm{MPa}$ in torsion bars, the 


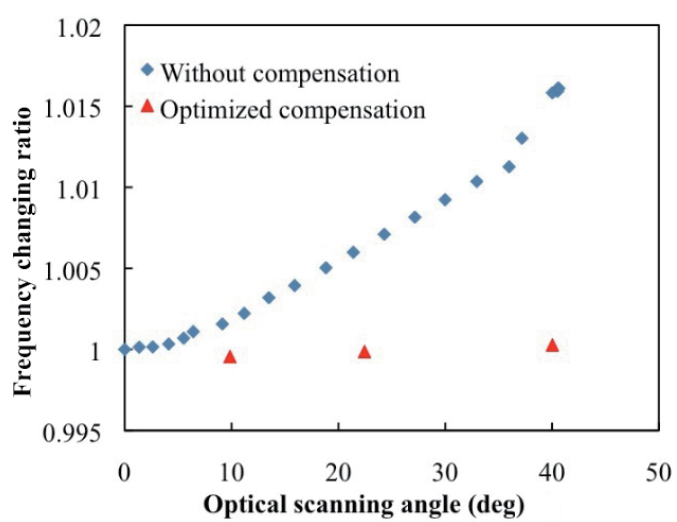

Fig. 10. (Color online) Resonant frequency shifts plotted against the scanning angle with and without frequency compensation.

corresponding spring constant decreases to $40 \%$ of its original value. The relationship between the frequency shift and the input dc power can be seen in Fig. 5 .

A nonlinear hard spring effect and the result of optimal compensation are shown in Fig. 10. The resonant frequency of the micromirror scanner is shifted higher than its original value by increasing the scanning angle. The tuning mechanism is driven to compensate this frequency shift. After the compensation is optimized, the frequency shift is suppressed from 1.5 to $0.05 \%$ at an optical scanning angle of $40^{\circ}$.

\section{Conclusion}

The design, fabrication and evaluation of a micromirror that can drive parametric amplification to increase the scanning amplitude are reported. It is demonstrated that the integrated bent beam actuator changes the spring constant of the torsional mode markedly with a ratio of $35 \%$ from the original spring constant. A novel scheme of the parametrically amplified scanning micromirror is proposed, and actually the amplitude of the mirror scanner driven in the torsional resonant mode can be amplified by electrothermal pumping. When the frequency of the pumping signal is double of the driving signal, the gain of the parametric amplification can be $9 \mathrm{~dB}$ of the maximum value. Additionally, the undesired resonant frequency shift based on the hard spring effect is suppressed from 1.5 to $0.05 \mathrm{~Hz}$ by using the bent beam actuator, which increases the driving scanning angle at a given driving power.

As the scanning angle increases, the resonant frequency shifts owing to the nonliner spring effect. The resonant frequency shift based on the spring tuner can be compensated for by applying additional dc power to the bent beam actuator to achieve a large scanning angle at a fixed driving frequency with a given driving power. The integrated electrothermal actuator can tune the torsional spring constant using a thermomechanically induced compressive stress. This actuation method capable of frequency tuning and parametric amplification can reduce the driving power of the scanning mirror, and also possibly improve the reliability. 


\section{Acknowledgements}

Part of this work was performed in the Micro/Nanomachining Research Education Center (MNC) of Tohoku University, as well as in the Junichi Nishizawa Memorial Research Center of Tohoku University. This work was supported in part by a Grant-in-Aid for Scientific Research from the Japanese Ministry of Education, Culture, Sports, Science and Technology of Japan, and also by Special Coordination Funds for Promoting Science and Technology, Formation of Innovation Center for Fusion of Advanced Technologies.

\section{References}

1 R. A. Conant, J.T. Nee, K. Lau, and R.S. Mueller: Proc. Tech. Dig. Solid-State Sensor and Actuator Workshop, 2000, p. 6.

2 L. J. Hombeck: Tech. Dig. Electron Devices Meeting (IEDM), 1993, p. 381.

3 J. Singh, J. H. S. Teo, Y. Xu, C. S. Premachandran, N. Chen, R. Kotlanka, M. Olivo, and C. J. R. Sheppard: J. Micromech. Microeng. 18 (2008) 025001.

4 P. D. Dobbelaere, K. Falta, L. Fan, S. Cloeckner, and S. Patra: IEEE Commun. Mag. 40 (2002) 88.

5 R. S. Muller and K. Y. Lau: Proc. IEEE 86 (1998) 1705.

6 H. Urey: Proc. SPIE 4773 (2002) 27.

7 D. Rugar and P. Grütter: Phys. Rev. Lett. 67 (1991) 699

8 T. Ono, H. Wakamatsu, and M. Esashi: J. Micromech. Microeng. 15 (2005) 2282.

9 D. W. Carr, S. Evoy, L. Sekaric, H. G. Craighead, and J. M. Parpia: Appl. Phys. Lett. 75 (1999) 920.

10 J. I. Lee, S. Park, Y. Eum, B. Jeong, and J. Kim: Microsys. Technol. 16 (2010) 979.

11 I. Veryeri and I. Basdogan: Microsyst. Technol. 16 (2010) 979.

12 M. Sasaki, S. Yuki, and K. Hane: IEEE Journal of Selected Topics in Quantum Electronics 13 (2007) 290.

13 L. Que, J. S. Parka, and Y. B. Gianchandani: J. Micromech. Microeng. 10 (2001) 247. 